\title{
STUDIES ON THE NEURO-HISTOLOGICAL CHANGES IN THE MEISSNER CORPUSCLE IN LEPROSY
}

\author{
By A. Paul. Jayaraj, F.r.m.s. \\ Central Food Technological Research Institute, Mysore \\ and \\ D. S. Chaudhury, m.B.B.S.
}

Gandhi Memorial Leprosy Foundation, T. Narasipur, Mysore

The Meissner corpuscle is one of the most complex cutaneous sensory receptors. In spite of extensive work on the role and the distribution of the nerve fibres in the Meissner corpuscle on normal individuals for nearly a century, there is still substantial controversy over the nature of the nerve termination and function of the corpuscle.

CAUNA (1956-B) conducted an interesting investigation on the nerve supply and their endings in Meissner corpuscles on material classified into various age and occupational groups (manual workers) in both sexes. He observed in old age the nerve endings are restricted to the distal end of the corpuscle, the rest of it consisting of twisted bundles of nerve fibres. In manual workers he observed that the nerve endings are reduced in number, but at the same time intracorpuscular nerve fibres showed neurofibrillar expansions.

The present work is an investigation of nerve supply and their changes in Meissner corpuscles in leprosy, since Meissner corpuscles act as a selective touch receptor and are primarily designed for tactile discrimination. An attempt has also been made to study the location of the bacilli in these receptors in relation to the neurofibrillary processes.

\section{Material and method}

Twenty-four pieces of skin were taken from the distal pad of the fingers from 24 patients showing typical lesions of leprosy, 14 from the lepromatous and 10 from tuberculoid cases. There were no visible lesions anywhere on the finger pads. Biopsies were also taken from the lesions of each patient to confirm histologically the type of lesion. Most of the specimens were taken from the back and a few from the forearm. Tissues were fixed in $10 \%$ neutral formalin and frozen sections were taken at 20 micron thickness and stained by the method described by Bala Subramanyan, Jayaraj and Gass (1954) for nerve fibres. 20 sections were taken from each specimen 
for this study. Remaining tissues were processed for paraffin sections. Sections were stained with hematoxylin and eosin and for acid fast bacilli by the method described by JaYARAJ (1955). As a control 3 specimens were taken from the distal pad of the fingers of manual workers.

\section{Results}

Changes in the Meissner corpuscle in lepromatous leprosy. The stem fibres ending in Meissner corpuscle show certain complexity and fragmentation. Most of the corpuscles receive single medullated nerve fibres which end with branching. Occasionally two medullated nerve fibres are seen ascending and ramifying in a most fragmented condition. The neural fragments are seen scattered all over the dermal papillac and they seem to line up with the neurofibrillary process of the corpuscle. In few cases Meissner corpuscles show the free fading filaments in the connective tissue. Bulbous fragments of the fibrillary structure are also found in the papillae, deep in the epithelium. In early lepromatous leprosy, the nerve endings in Meissner corpuscles undergo less damage compared to advanced lepromatous leprosy. Most of the corpuscles in this stage of leprosy show normal neurofibrillary structure.

Paraffin sections stained for acid fast bacilli show that the bacilli are situated alongside the visible fibrillary ramification in the Meissner corpuscles. They are found abundant in early lepromatous leprosy. In advanced lepromatous leprosy the bacilli are found less in number.

Changes in tuberculoid leprosy. The degenerated nerve fibres ascend in a most difficult course towards the papillary region where they usually end as naked fading filaments. Most of the fibres break up into several segments which disintegrate in the illformed corpuscle. In a few specimens complete destruction of Meissner corpuscles are noticed. Several neural fragments are found in corpuscles and they are highly macerated and are not connected to the stem fibre. The epidermis is much flattened and the papillary regions are collapsed.

The paraffin sections stained for acid fast bacilli have not shown the presence of bacilli.

Changes in Manual Workers. The ascending stem fibres reach the papillary region without any changes compared to normal individuals. The ramification of the fine nerve filaments show expansion in the end bulbs. The papillary regions are slightly compressed.

\section{Discussion}

Meissner corpuscles contain a series of nerve fibres. The stem fibres ascend within the capsule and branch in layers in between the cells parallel to the surface of the capsule and to their stem fibres. 


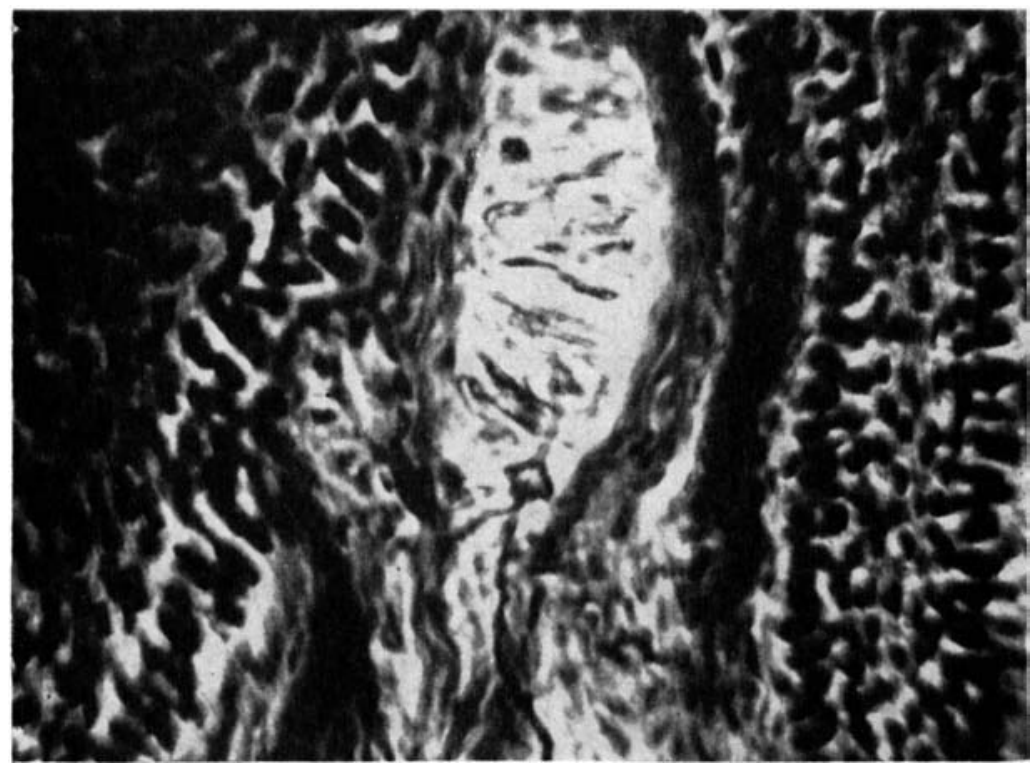

Fig. 1. A Meissner corpuscle in the dermal papillae showing normal neurofibrillary coiling with little of terminal thickening in the corspucle. Distal pad of middle finger, male, 30 years, manual ivorker. $x 40$.

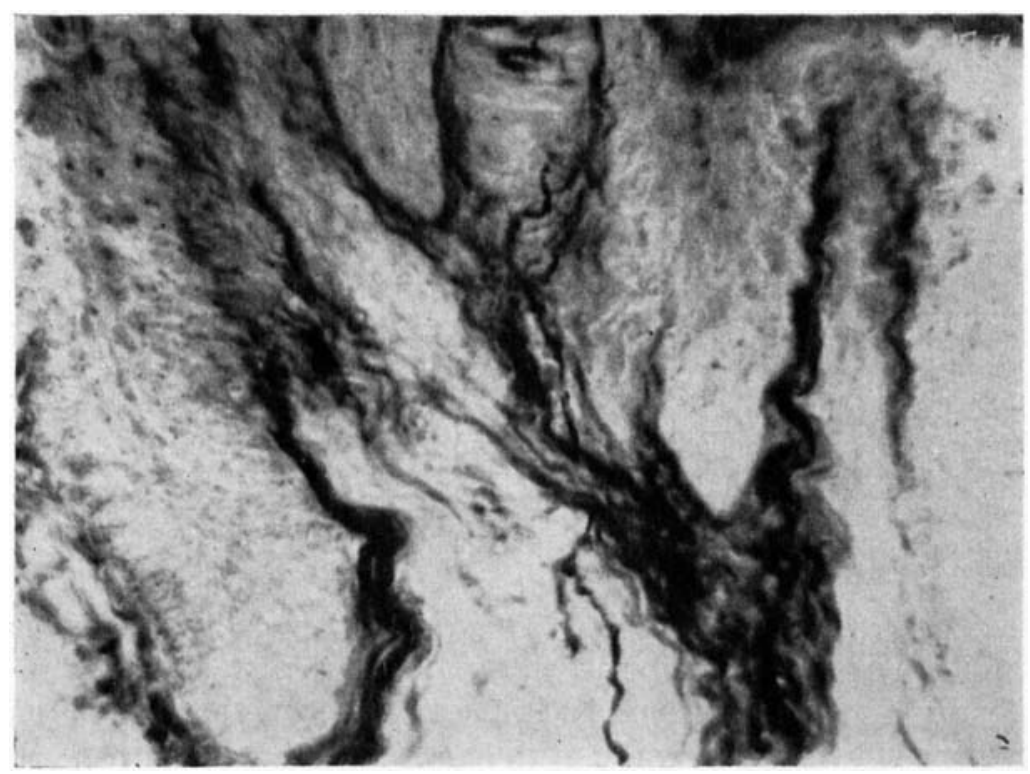

Fig. 2. A Meissner corpuscle in the dermal papillae showing a single myelinated stem fibre ascending from the subcutaneous tissue to the corpuscle where it branches as fading nerve filament. On the top of the corpuscle thickened fibrillary structure is seen disconnected to the stem fibre. Distal pad of the ring finger, male, 28 years, advanced lepromatous leprosy. $x 400$. 


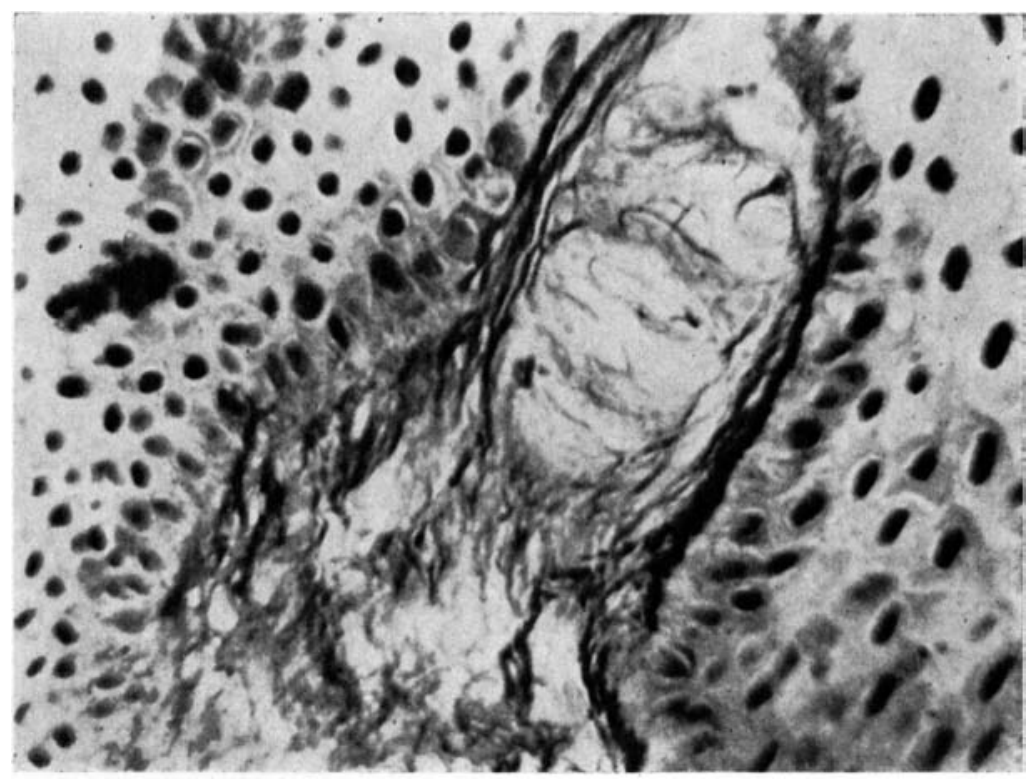

Fig. 3. A Meissner corpuscle in the dermal papillae showing a single stem fibre ascending into the corpuscle where it ramifies with partially fading nerve filaments without much structural alteration. Distal pad of the middle finger, male, 30 years, advanced lepromatous leprosy. x400.

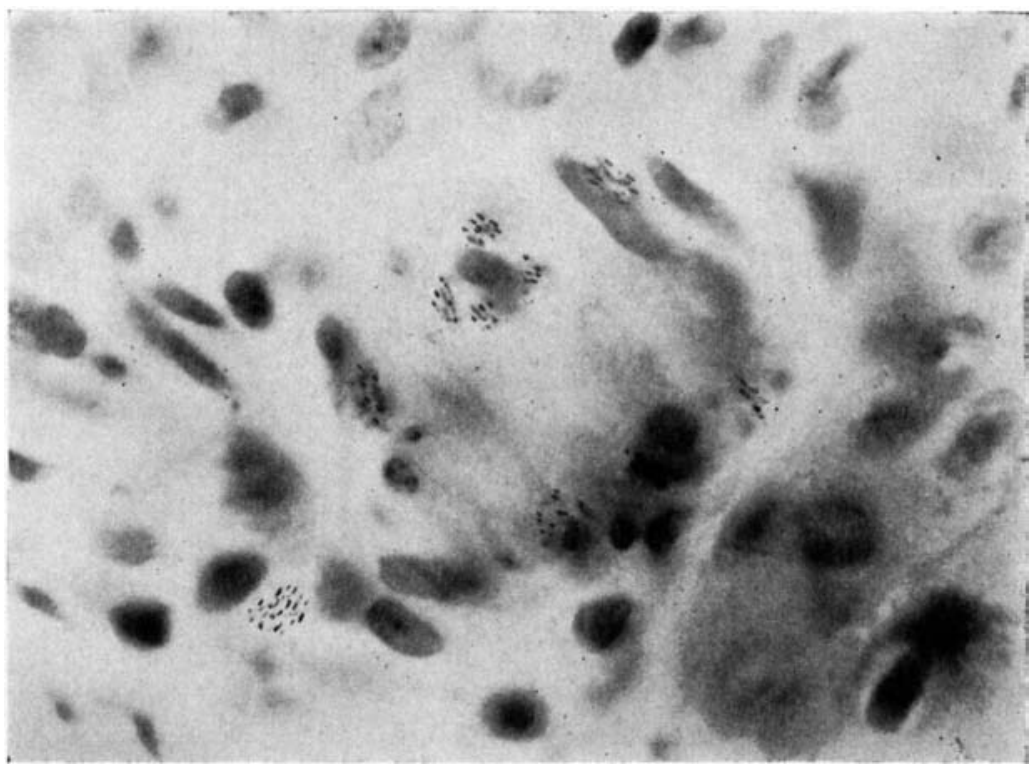

Fig. 4. Meissner corpuscle in the dermal papillae shows the clumps of bacilli situated alongside the neurofibrillary processes in the corpuscle. Paraffin section stained for acid-fast bacilli. Distal pad of the ring finger, male, 25 years. Early lepromatous leprosy. x 900 . 


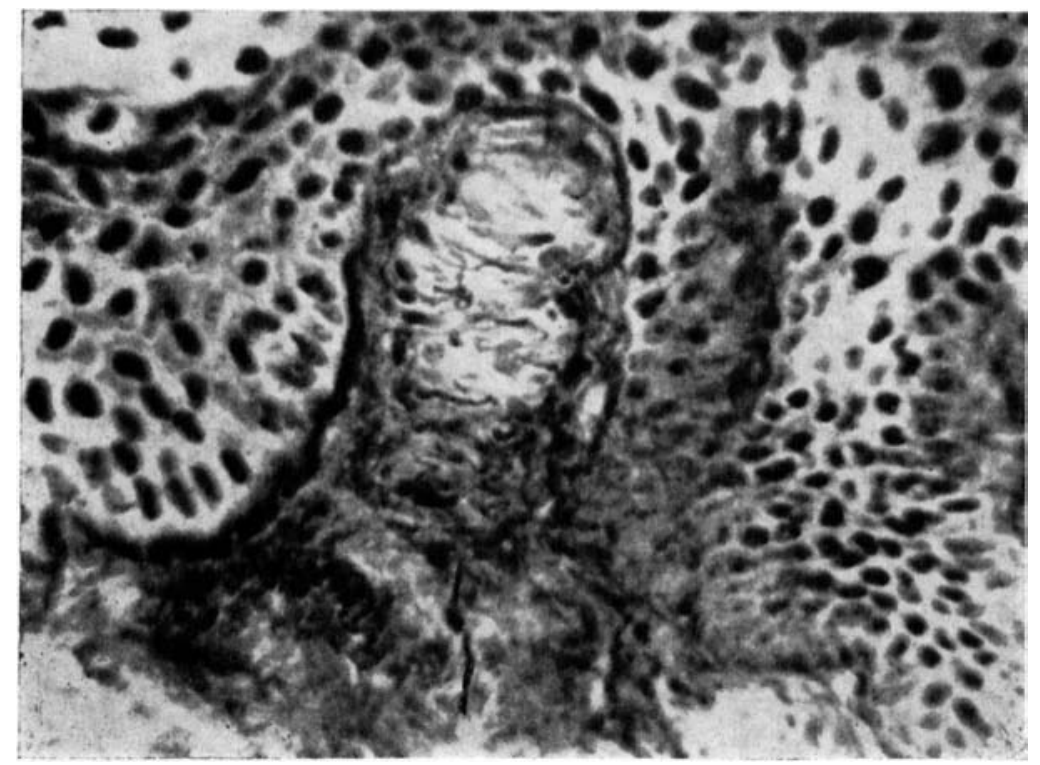

Fig. 5. Meissner corpuscle in the dermal papillae showing a single ascending nerve fibre ramifying in the corpuscle with partially fading filaments. Several neural segments are seen. Distal pad of the ring finger. Male, 36 years. Early lepromatous leprosy. $\mathrm{x} 400$.

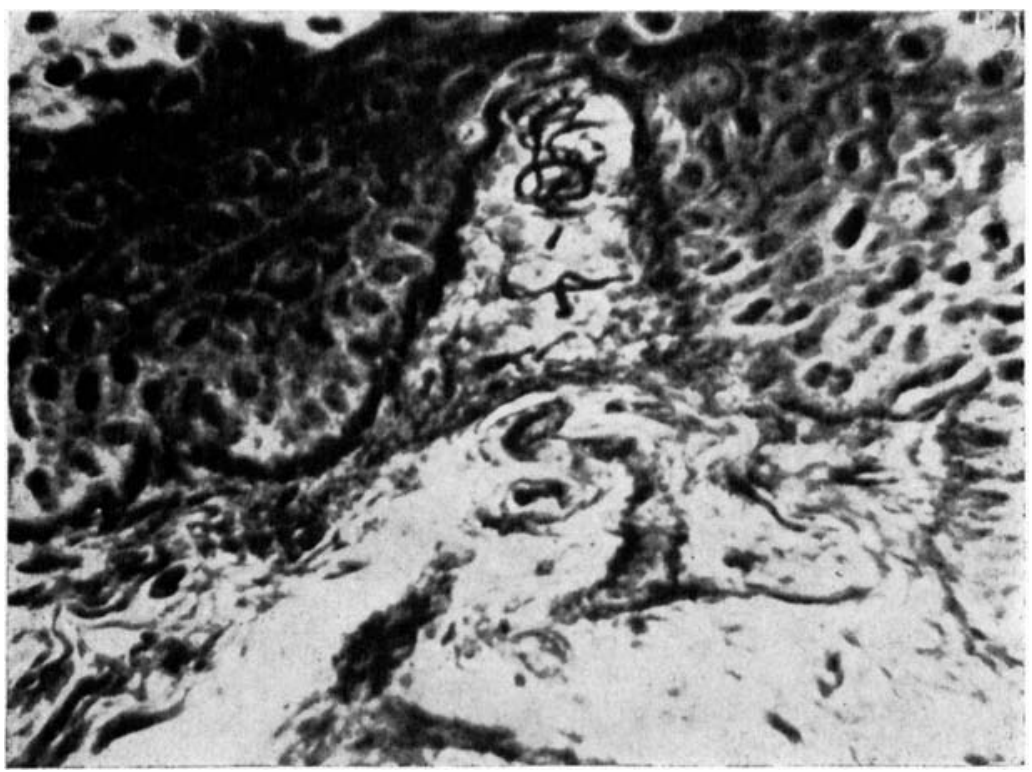

Fig. 6. Meissner corpuscle showing a myelinated single fibre ascending right up to the dermal papillae with limited coiling. The whole process looks myelinated. Distal pad of the ring finger. Male, 28 years. Lepromatous leprosy. x400. 


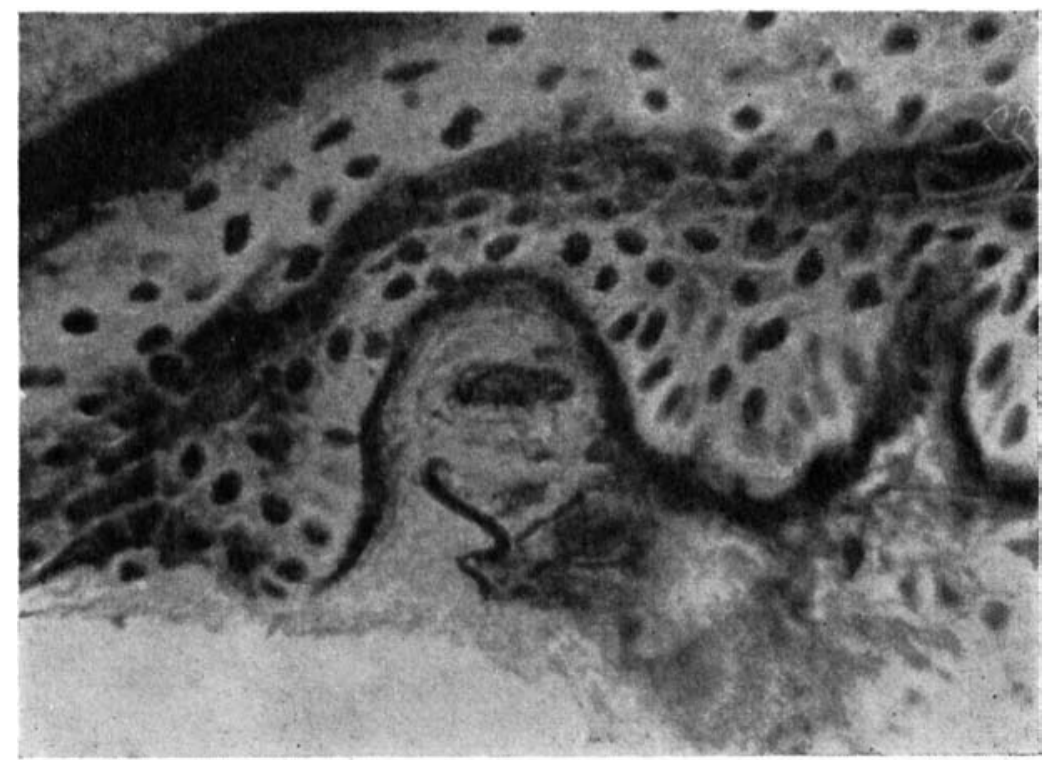

Fig. 7. Meissner corpuscle showing two myelinated nerve fibres ascending into the flattened dermal papillae and ramifying with faded nerve filament. A thickened neural element is seen in the middle of the corpuscle unconnected to the stem fibre. Distal pad of the middle finger. Male, 37 years. Lepromatous leprosy. $\mathrm{x} 400$.

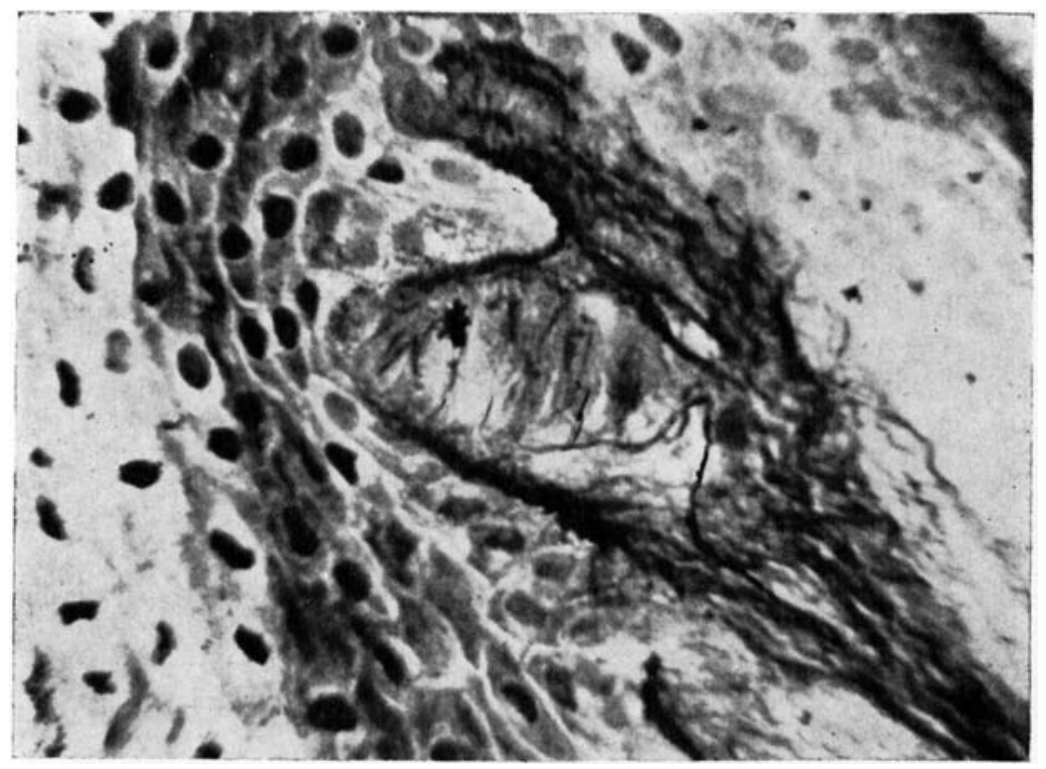

Fig. 8. Meissner corpuscle showing a single stem fibre ascending into the corpuscle and branching with number of neural segments and feeding filaments. Distal pad of the middle finger. Male, 28 years. Tuberculoid leprosy. x400. 


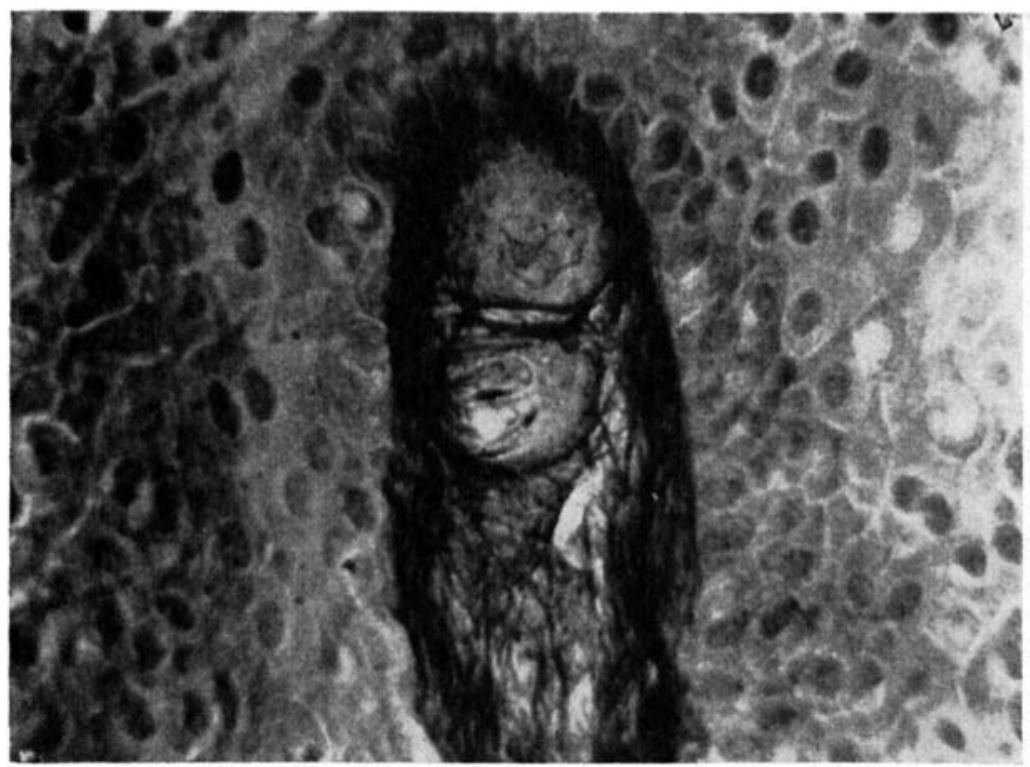

Fig. 9. Meissner corpuscle in the dermal papillae showing the corpuscle embedded in the connective tissue elements and the neural fragments situated in the centre of the lobulated corpuscle. Distal pad of the ring finger. Male, 30 years. Tuberculoid leprosy. $\mathrm{x} 400$.

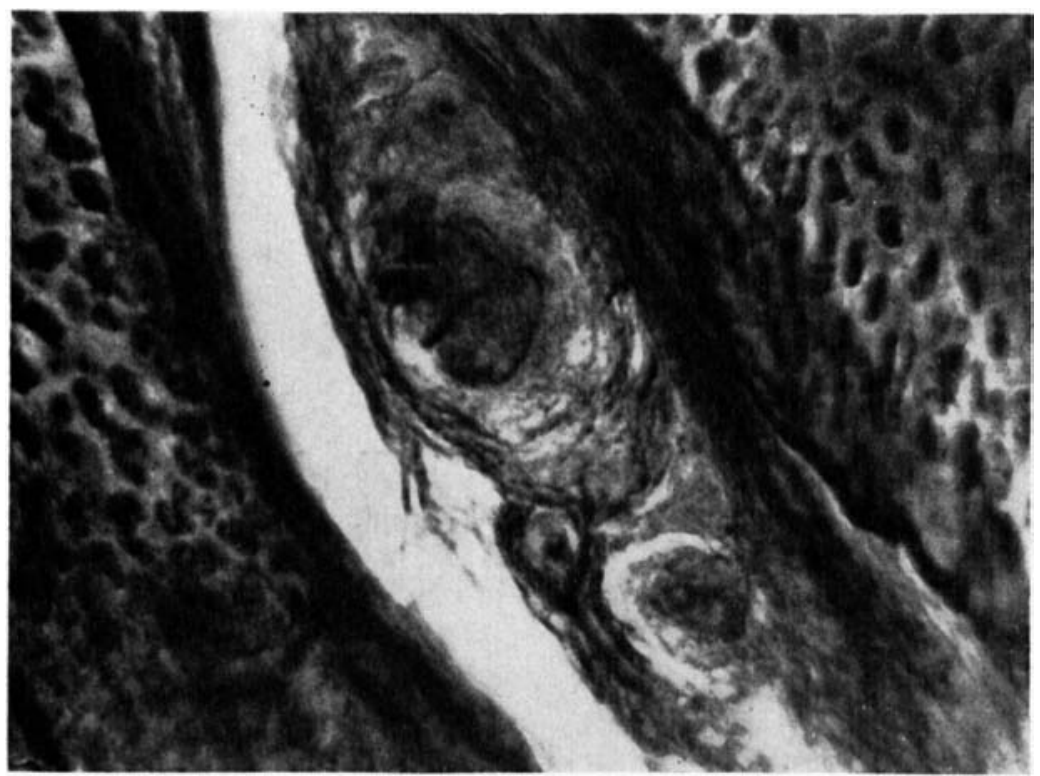

Fig. 10. Meissner corpuscle in the dermal papillae showing the neural mass in the centre of the corpuscle with structural collapse. The two stem fibres which reach the corpuscle show degenerated changes and are not connected to the fine neural elements situated in the centre of the corpuscle. Distal pad of the middle finger. Male, 22 years. Tuberculoid. $x 400$. 


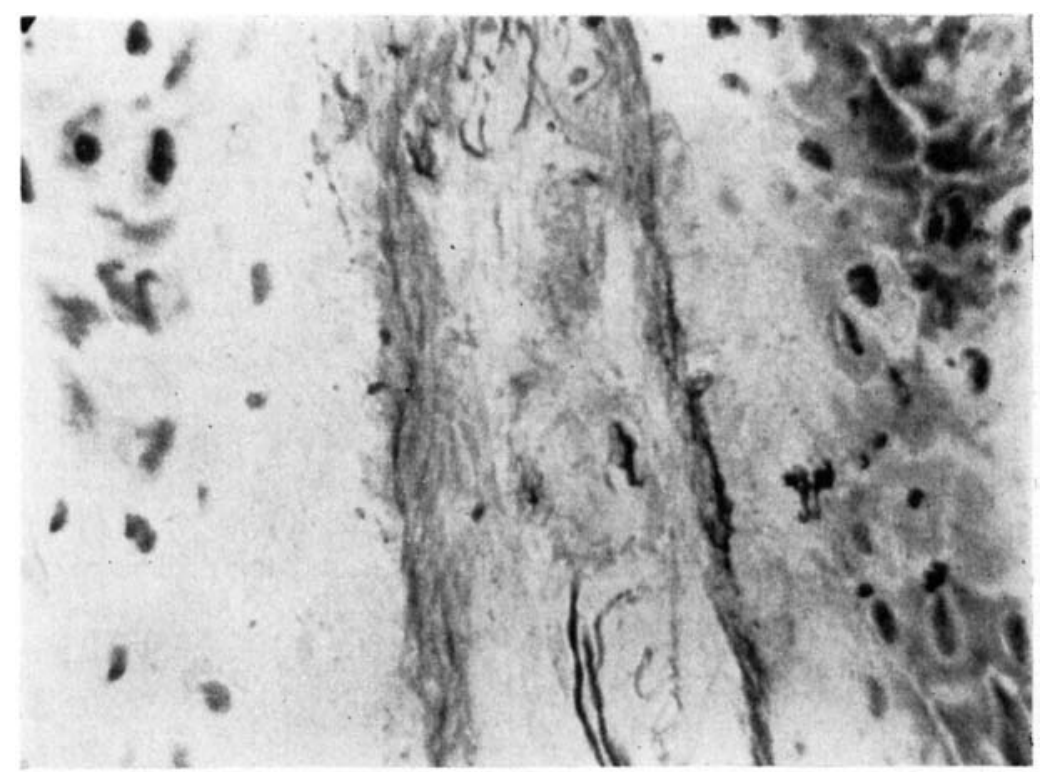

Fig. 11. Two stem fibres are seen ascending in the dermal papillae. The ramifying neural filaments are destroyed and a few nerve filaments are seen deep in the dermal papillae unconnected to the stem fibres. Distal pad of the ring finger. Male, 30 years. Tuberculoid. x400.

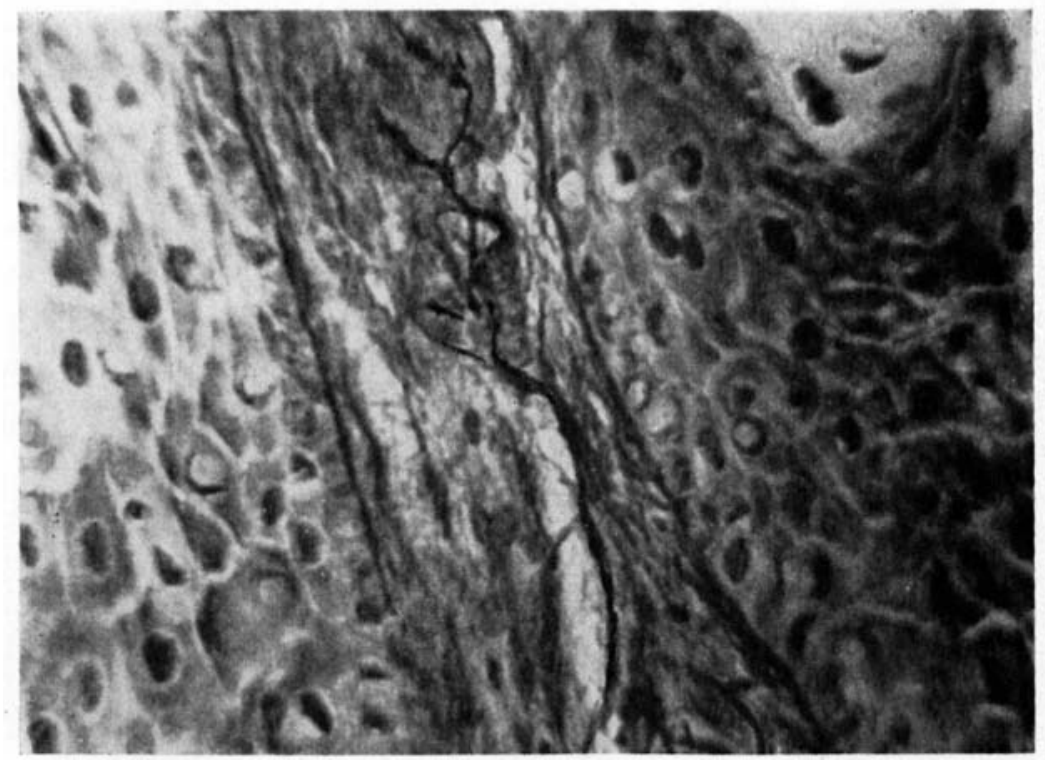

Fig. 12. A single nerve fibre is seen ascending into dermal papillae with irregular branching and embedded into the connective tissue elements. Distal pad of the ring finger. Male, 40 years. Tuberculoid. $x 400$. 
Direct pressure will produce stimulation to all these fibres. WEDDI:LL (1941a) showed the pattern of cutaneous innervation in relation to cutaneous sensibility and brought out the clinical significance of the pattern of cutaneous innervation. He demonstrated that the density of innervation of skin varies from place to place and that tactile acuity is dependant upon both the numbers of compact encapsulated nerve endings and the number of pre-terminal nerve fibres per unit field serving them. He further observed that in hairy skin, there are normally no receptor bodies like Meissner corpuscle other than the nerve endings related to hairs. In non-hairy areas, Meissner corpuscles and their functional status determine the tactile acuity of the concerned area.

In leprosy the changes in Meissner corpuscles are of considerable interest. Alteration of the Meissner corpuscles in manual workers has been reported by CAUNA (1956 B). However there are no reports in detail in the literature on the changes of Meissner corpuscles in leprosy. In recent years a considerable amount of work has been done on nerve changes in leprosy. Gass and Balsubramanyan (1954) studied the damage of nerves in different kinds of leprosy lesions. Balsubramanyan, JayaraJ and Gass (1954) demonstrated the acid fast bacilli in the myelinated nerve fibres. Gault, JayaraJ and Gass (1955) demonstrated the bacillus containing capillaries and axons by treatment for alkaline and acid phosphatases. They observed that the bulbous swelling of axons are filled up with bacilli. KHANOLKaR (1951) brought out the theory that the bacilli invade the fine nerve and travel into the axons. MUKhERJ et $a$ in a study by the acid phosphatase method described by Gault, JayaraJ and Gass (1955), observed parenchymatous degeneration of fibres in lepromatous leprosy and thought it to be possibly of toxic origin. They further observed that the changes in tuberculoid leprosy start with perineural infiltration in the finer nerves near the epidermis. The compact perineural infiltration, they found, penetrated into the thicker nerve and brought about fragmentation of fibres which lead to Wallerian degeneration. In the lepromatous lesions, they found no fragmentation of fibres. They observed that location of bacilli in the cutaneous nerves was mainly in the inter-fibre spaces, but in the lepromatous lesions they were occasionally encountered in the different parts of a nerve fibre, such as myelin sheath, Schwann sheath, Schwann cell and faintly stained axon. JAYARAJ and CHAUDHURY (1959) reported the existence of nerve fibres even in advanced lepromatous leprosy in the most superficial layers of the epithelial and sub-epitheilal regions. The present observation shows that in Meissner corpuscles in early lepromatous leprosy the bacilli invade the whole processes of the corpuscles when the corpuscle maintains its normal ramification of fine axons. In the later stages the bacilli are not found in these corpuscles and are found more in stem fibres. However the 
structural ramification of the fine nerve fibres in Meissner corpuscles is always maintained in lepromatous leprosy without much alteration and damage. In tuberculoid leprosy the stem fibres that enter into the corpuscles show more segmentation and they do not branch and ramify into the capsule. The continuity of the fine fibres is not seen. It looks as if the corpuscle is completely damaged in this type of leprosy.

\section{Summary}

1. Biopsies from distal pad of the fingers from 24 leprosy patients comprising 14 lepromatous and 10 tuberculoid were studied hy cytological and nerve staining methods.

2. It was found that the terminal fibres in Meissner corpuscles undergo characteristic changes in leprosy. In early lepromatous leprosy abundant hacilli were found alongside of the neuro-fibrillary ramification and the corpuscles look almost normal. Whereas in advanced lepromatous leprosy, bacilli were not found in Meissner's corpuscles and the corpuscle was found slightly damaged.

3. In tuberculoid leprosy the fading filaments are commonly found in the corpuscle. The papilla that occupies the corpuscle is compressed causing severe damage to the corpuscle. The ascending stem fibres which reach the papillae undergo severe damage by way of fragmentation leaving several neural filaments far away in the papillary region not connected to the stem fibre.

4. It is generally assumed that the receptor mechanism does play a part in alteration of sensory modalities more in tuberculoid type of leprosy and less in lepromatous leprosy. There is a tendency for regaining the structural and functional status of Meissner corpuscles in lepromatous leprosy when the disease process is arrested. The possibilities in regaining the functional and structural status in tuberculoid leprosy are far less.

\section{Acknowledgments}

Our grateful thanks are due to Dr. V. Subrahmanyan, D.sc., F.R.I.C., F.N.I., Director, Central Food Technological Research Institute, for kindly offering all facilities and encouragements to this investigation and to Dr. R. V. Wardekar, B.sc., M.D., Secretary, Gandhi Memorial Leprosy Foundation, for kind permission to do this investigation. We are also indebted to Dr. M. Swaminathan, D.sc., F.N.I., Assistant Director, Central Food Technological Research Institute, Mysore, for his kind and valued encouragements. 
We express our gratitude to Mr. K. V. Achyuta Rao, for his aid with the photographic work.

\section{References}

1. Cauna, N., (1956 B), "Nerve Supply and Nerve Endings in Meissner's Corpuscles", Amer. J. Anat., 99, 315-350.

2. Weddell, G., (1941 a), "The Pattern of Cutaneous Innervation in Relation to Cutaneous Sensibility", J. Anat., London, 75, 346.

3. Balasubramanyan, M., Jayaraj, A. P., and Gass, H. H., (1954), "An Improved Histological Method for the Examination of Cutaneous Nerve in Leprosy", Leprosy Review, 15, 83-86.

4. JayaraJ, A. P., (1955), "Periodic Acid in the Staining of Acid-fast Bacilli in Tissue Section", J. of the Anat. Soc. of India, Vol. 4, 41-42.

5. Gass, H. H., and Balusubramanyan, M., (1954), "Change in Cutaneous Nerves in Leprosy", Int. J. Lep., 22, 31-40.

6. Gault, E. W., Jayaraj, A. P., and Gass, H. H., (1955), "The Application of Histochemical Methods in the Study of the Skin in Leprosy", Int. J. Lep., 23, (1955), 2.

7. KhanOlkaR, V. R., (1951), "Studies in the Histology of Early Lesions in Leprosy", Indian Council of Medical Research, special report series No. 19 1951, P-18.

8. Jayaraj, A. P., and Chaudhury, D. S., (1959). Presented in the All India Leprosy Conference and will be published in Leprosy in India.

9. MukherJi, N., and Ghosal, P., (1957), "Study of Cutaneous Nerves in Leprosy by Acid Phosphatase Method", Leprosy in India, Vol. XXIX, No. 1, pp.3-7. 